\title{
Variabilidade do Fenótipo de Pacientes Com Síndrome de Noonan Com e Sem Mutaçōes no Gene PTPN1 1
}

\section{artigo original}

\author{
LIZE V. FERREIRA \\ SILVIA A.L. SOUZA \\ LUCIANA R. MONTENEGRO \\ IVO J.P. ARNHOLD \\ titania Pasqualini \\ JuAN Jorge HeinRICH \\ ana Claudia Keselman \\ BeRENICE B. Mendonça \\ AleXANDER A.L. Jorge
}

Laboratório de Hormônios e Genética Molecular - LIM/42

(LVF, SALS, LRM, IJPA, BBM \&

AALJ), Disciplina de

Endocrinologia do

Departamento de Clínica

Médica do Hospital das

Clínicas, da Faculdade de Medicina da Universidade de São Paulo, SP, Brasil; Sección Endocrinología, Crecimiento y Desarrollo, Departamento de Pediatría (TP), Hospital Italiano de Buenos Aires, e Division de Endocrinologia (JJH \& ACK),

Hospital de Niños Ricardo Gutierrez, CEDIE, Buenos Aires, Argentina.

Recebido em 01/06/06 Revisado em 05/10/06 Aceito em 09/10/06

\section{RESUMO}

Introdução: Aproximadamente $50 \%$ dos pacientes com síndrome de Noonan (SN) apresentam mutações em heterozigose no gene PTPN11. Objetivo: Avaliar a freqüência de mutações no PTPN11 em pacientes com SN e analisar a correlação fenótipo-genótipo. Pacientes: 33 pacientes com SN. Método: Extração de DNA de leucócitos periféricos e seqüenciamento dos 15 exons do PTPN11. Resultados: Nove diferentes mutações missense no PTPN11, incluindo a mutação $\mathrm{P} 491 \mathrm{H}$, ainda não descrita, foram encontradas em 16 dos 33 pacientes. As características clínicas mais freqüentes dos pacientes com SN foram: pavilhão auricular com rotação incompleta e espessamento da helix $(85 \%)$, baixa estatura (79\%), prega cervical $(77 \%)$ e criptorquidismo nos meninos (60\%). O Z da altura foi de $-2,7 \pm 1,2$ e o do IMC foi de $-1 \pm 1,4$. Os pacientes com mutação no PTPN11 apresentaram maior freqüência de estenose pulmonar do que os pacientes sem mutação (38\% vs. $6 \%, p<0,05)$. Pacientes com ou sem mutação no PTPN11 não diferiram em relação à média do $Z$ da altura, Z do IMC, freqüência de alterações torácicas, características faciais, criptorquidia, retardo mental, dificuldade de aprendizado, pico de $\mathrm{GH}$ ao teste de estímulo e Z de IGF-1 ou IGFBP-3. Conclusão: Identificamos mutações no PTPN11 em 48,5\% dos pacientes com $\mathrm{SN}$, os quais apresentaram maior freqüência de estenose pulmonar. (Arq Bras Endocrinol Metab 2007;51/3:450-456)

Descritores: Síndrome de Noonan; Mutações; Gene PTPN11; Genótipo e fenótipo

\section{ABSTRACT}

Phenotype Variability in Noonan Syndrome Patients With and Without PTPN11 Mutation.

Introduction: Around $50 \%$ of Noonan syndrome (NS) patients present heterozygous mutations in the PTPN11 gene. Aim: To evaluate the frequency of mutations in the PTPN11 in patients with NS, and perform phenotype-genotype correlation. Patients: 33 NS patients (23 males). Methods: DNA was extracted from peripheral blood leukocytes, and all 15 PTPN11 exons were directly sequenced. Results: Nine different missense mutations, including the novel $\mathrm{P} 491 \mathrm{H}$, were found in 16 of $33 \mathrm{NS}$ patients. The most frequently observed features in NS patients were posteriorly rotated ears with thick helix $(85 \%)$, short stature $(79 \%)$, webbed neck $(77 \%)$ and cryptorchidism $(60 \%)$ in boys. The mean height SDS was $-2.7 \pm 1.2$ and BMI SDS was $-1 \pm 1.4$. Patients with PTPN11 mutations presented a higher incidence of pulmonary stenosis than patients without mutations $(38 \%$ vs. $6 \%, p<0.05)$. Patients with and without mutations did not present differences regarding height SDS, BMI SDS, frequency of thorax deformity, facial characteristics, cryptorchidism, mental retardation, learning disabilities, GH peak at stimulation test and IGF-1 or IGFBP-3 SDS. Conclusion: We identified missense mutations in $48.5 \%$ of the NS patients. There was a positive correlation between the presence of PTPN11 mutations and pulmonary stenosis frequency in NS patients. (Arq Bras Endocrinol Metab 2007;51/3:450-456)

Keywords: Noonan syndrome; Mutations; PTPN11 gene; Genotype and phenotype 
A s Síndrome de NoONAn (SN, OMIM 163950) é uma doença de herança autossômica dominante, caracterizada por alterações dismórficas com grande variedade de expressão fenotípica. A SN ocorre numa freqüência estimada de 1:2500 - 1:1000 nascidos vivos, afetando igualmente ambos os sexos (1). Classicamente, os pacientes apresentam baixa estatura proporcionada, fácies característica (face triangular, hipertelorismo, fenda palpebral voltada para baixo, epicanto, ponte nasal baixa e larga, orelhas de implantação baixa com hélice espessada, pescoço alado), deformidade torácica (pectus excavatum e/ou carinatum, hipertelorismo mamilar), cardiopatia congênita (estenose valvar pulmonar, cardiomiopatia hipertrófica), além de outras alterações observadas com menor freqüência: déficit visual ou auditivo, discreto retardo do desenvolvimento neuropsicomotor, alterações do aprendizado, hepatoesplenomegalia, alterações de coagulação, distúrbios digestivos e criptorquidia (2). A baixa estatura é uma característica marcante dessa síndrome, com escore de desvio-padrão da altura final ( $\mathrm{Z}$ da altura final) de $-2,5$ para o sexo masculino e de $-2,1$ para o sexo feminino (3). O diagnóstico clínico da $\mathrm{SN}$ é baseado em um sistema de pontuação, com critérios maiores e menores, proposto por van de Burgt em 1994 (4) (tabela 1). O diagnóstico da SN baseado apenas nas características clínicas pode ser difícil, especialmente na ausência de cardiopatia ou em idade mais avançada, quando as características faciais se tornam menos evidentes.
Em 2001, mutações no gene PTPN11 (Protein tyrosinephosphatase non-receptor 11 - OMIM 163950) foram identificadas por Tartaglia e col. em pacientes com $\mathrm{SN}(5)$, permitindo, pela primeira vez, a confirmação molecular de casos de $\mathrm{SN}$ diagnosticados clinicamente. Os estudos publicados até a presente data identificaram mutações do PTPN11 em $40 \%$ dos pacientes com SN, variando de 26 a 37\% nos casos esporádicos e de 50 a 100\%, nos familiares. Os exons $3,7,8$ e 13 são regiões hot spot, concentrando $86,7 \%$ das mutações descritas (6).

O gene PTPNII codifica a proteína tirosinofosfatase SHP-2 (Src Homology region2domain Phosphatase 2 - OMIM 176876), que se caracteriza por apresentar dois domínios SH2 (N-SH2 e C-SH2) pelos quais a SHP-2 reconhece e se liga a sítios de tirosina fosforilado de receptores ou de proteínas adaptadoras que fazem parte da cascata de sinalização de receptores tipo tirosinaquinase ou de citoquinas. Além dos domínios SH2, a SHP-2 apresenta um domínio catalítico (PTP) que é responsável pela sua capacidade de desfosforilar sítios de tirosina fosforiladas e, também, em sua porção carbóxi terminal possui um resíduo de tirosina que, ao ser fosforilada, confere à SHP-2 a capacidade de também atuar como uma proteína acopladora da cascata de sinalização de diversos receptores. A SHP2 , na sua forma inativa, tem sua atividade catalítica bloqueada por uma interação do domínio N-SH2 com o domínio PTP, quando a SHP-2 liga-se a uma

Tabela 1. Critérios de van der Burgt e cols. para diagnóstico da síndrome de Noonan (4).

\begin{tabular}{|c|c|c|c|}
\hline \multirow{2}{*}{\multicolumn{2}{|c|}{ Características }} & \multicolumn{2}{|c|}{ Critérios } \\
\hline & & Maiores & Menores \\
\hline 1. Faciais & & Típica & Sugestiva \\
\hline 2. Cardíac & & $\begin{array}{l}\text { Estenose valvar pulmonar } \\
\text { Miocardiopatia hipertrófica }\end{array}$ & Outras \\
\hline 3. Altura & & $<3^{\circ}$ percentil & $<10^{\circ}$ percentil \\
\hline 4. Torácic & & $\begin{array}{c}\text { Pectus carinatum e/ou } \\
\text { excavatum }\end{array}$ & Alargado \\
\hline 5. História & Familiar & $\begin{array}{l}\text { Parente de } 1^{\circ} \text { grau com } \\
\text { diagnóstico de } \mathrm{SN}\end{array}$ & $\begin{array}{l}\text { Parente de } 1^{\circ} \text { grau } \\
\text { sugestivo de } \mathrm{SN}\end{array}$ \\
\hline 6. Outros & $\begin{array}{l}\text { Retardo mental } \\
\text { Criptorquidia } \\
\text { Displasia linfática }\end{array}$ & Todos os 3 & Qualquer \\
\hline
\end{tabular}

Face típica: face triangular, fenda palpebral oblíqua com o ângulo esterno voltado para baixo, hipertelorismo ocular, ptose palpebral, pavilhão auricular malformado e de implantação baixa, micrognatia, pescoço alado.

Diagnóstico de SN: Face típica + 1 outro critério maior ou 2 menores; Face sugestiva +2 outros critérios maiores ou 3 menores. 
tirosina fosforilada através dos seus domínios SH2 ocorre uma mudança conformacional da sua estrutura, que desfaz a interação entre os domínios N-SH2 e PTP, acarretando um aumento da sua atividade tirosina fosfatase. As mutações do gene PTPNI1, associadas com a $S \mathrm{~N}$, aumentam a atividade catalítica da SHP-2 por diminuírem ou eliminarem a interação dos domínios N-SH2 e PTP que mantêm a SHP-2 em seu estado inativo (6).

$\mathrm{Na}$ célula, a SHP-2 está envolvida no mecanismo fisiológico de interrupção do sinal dos receptores tirosinoquinases e dos receptores de citoquinas, como, por exemplo, o receptor do GH (GHR) (7). Esses receptores precisam ser fosforilados para iniciarem a transdução do sinal intracelular, e a desfosforilação realizada pela SHP-2 interrompe esse sinal $(7,8)$. Porém, este aumento na atividade tirosina fosfatase da SHP-2, causado pelas mutações no PTPNII associadas à $\mathrm{SN}$, também está associado a um aumento da sinalização intracelular via Ras e, por essa via, atua na gênese de doenças mieloproliferativas, principalmente da leucemia mielomonocítica juvenil (MIM 607785) (6).

Nosso objetivo foi estudar o gene PTPNII em um grupo de pacientes com o diagnóstico clínico da síndrome de Noonan e analisar a correlação entre o genótipo e o fenótipo.

\section{CASUÍSTICA E MÉTODOS}

\section{Pacientes}

O trabalho foi aprovado pela comissão de Ética do Hospital das Clínicas da Faculdade de Medicina de São Paulo, os pacientes ou seus responsáveis legais foram devidamente informados sobre o estudo e deram seu consentimento para a realização do mesmo. Estudamos 33 pacientes com SN (23 do sexo masculino e 10 do sexo feminino), diagnosticados segundo os critérios clínicos de van der Burgt (4) (tabela 1).

\section{Avaliação hormonal}

Os pacientes foram avaliados quanto à integridade do eixo GH/IGF-1 utilizando testes de liberação do GH (teste da clonidina) e dosagem basal de IGF-1 e IGFBP-3. O GH foi dosado por método imunofluorométrico com anticorpos monoclonais (AutoDELFIA, Wallac, Turku, Finland). Os ensaios foram normatizados com o padrão internacional $80 / 505$ da OMS e o coeficiente de variação interensaio é menor que $6 \%$ nas concentrações entre $0,1-38 \mathrm{ng} / \mathrm{ml}$. Valores de GH acima de $3,3 \mathrm{ng} / \mathrm{ml}$ excluíram a deficiência de hormônio de crescimento (9). A concentração de IGF-1 foi determinada por RIE após extração com etanol (DSL, Webster, TX, USA), e a de IGFBP-3 foi determinada por método imunorradiométrico (DSL, Webster, TX, USA). O coeficiente de variação interensaio foi menor que $10 \%$ para níveis de IGF-1 entre $18-500 \mathrm{mg} / \mathrm{l}$ e para níveis de IGFBP3 entre $0,14-12 \mathrm{mg} / \mathrm{dl}$.

\section{Estudo molecular}

DNA genômico foi isolado de leucócitos periféricos. O gene PTPN11 foi estudado por amplificação dos exons 1-15 utilizando primers intrônicos específicos (as seqüências dos primers e os protocolos de amplificação estão disponíveis se requisitados). Os produtos de PCR foram submetidos a seqüenciamento direto.

\section{Estatística}

As variáveis foram expressas em média \pm desvio-padrão. As variáveis qualitativas foram comparadas pelo teste de Fisher. Para a comparação das características quantitativas, utilizamos os testes t de Student ou Kruskal-Wallis. Para toda análise, consideramos significante valor de $\mathrm{p}<0,05$.

\section{RESULTADOS}

\section{Dados clínicos}

Dos 33 pacientes com SN, 2 casos (6,1\%) possuíam história familiar de $\mathrm{SN}$ e 31 casos eram isolados. A idade média na avaliação inicial foi de $10,7 \pm 4,5$ anos. $\mathrm{O}$ escore do desvio-padrão da altura ( $\mathrm{Z}$ da altura) médio foi de $-2,7 \pm 1,2$, significativamente inferior à altura dos pais ( $\mathrm{Z}$ da altura-alvo de $-0,7 \pm 1,0)$. Vinte e seis crianças apresentavam baixa estatura, definido por $\mathrm{Z}$ da altura $<-2$. Sete crianças apresentavam altura dentro da normalidade, porém sempre abaixo da média para idade e sexo. Foi observada uma diminuição significativa do peso, expresso como escore de desvio-padrão do IMC, em comparação à referência (Z IMC $=-1 \pm 1,4 ; \mathrm{p}<0,001)$. A maioria dos pacientes apresentou puberdade de início tardio e a idade média do início dos caracteres sexuais secundários foi de $13 \pm$ 1,8 anos nos meninos $(n=15)$ e de $12 \pm 1,5$ anos nas meninas $(n=6)$.

Os sinais clínicos associados com a SN mais freqüentemente observados em nossos pacientes foram: alterações do pavilhão auricular (implantação baixa e/ou espessamento da hélice) em $85 \%$ dos pacientes, baixa estatura em $79 \%$ e pescoço alado em $77 \%$. Mais da metade dos pacientes apresentava face típica (60\%). Nos pacientes do sexo masculino, foi também constatada criptorquidia em $60 \%$ dos casos. Metade dos nossos pacientes apresentava malformações cardíacas, sendo 9 pacientes (28\%) portadores de cardiopatia grave $(6 \mathrm{com}$ estenose pulmonar, $1 \mathrm{com}$ estenose pulmonar e CIA, l com miocardiopatia hipertrófica isolada e outro associada a CIA); 6 (18\%) apresentavam lesões cardíacas leves sem repercussão clínica 
significante: valva mitral espessada (3 pacientes), prolapso de valva mitral, valva tricúspide espessada e ectasia de aorta torácica, respectivamente em l paciente cada.

\section{Dados laboratoriais}

A secreção de GH foi avaliada em 27 pacientes $(81,8 \%)$ diretamente através de teste de estímulo (21 pacientes) e/ou indiretamente pela dosagem de IGFl e/ou IGFBP-3 (23 pacientes). Todos os pacientes com SN submetidos ao teste de estímulo com clonidina apresentaram resposta de $\mathrm{GH}$ que permitiu afastar a deficiência desse hormônio (pico de GH nos testes $13,0 \pm 6,4 \mathrm{ng} / \mathrm{ml})$. A concentração média de IGF- 1 foi de $-1,6 \pm 1,9 \mathrm{DP}(36,4 \%$ abaixo de $-2 \mathrm{DP})$, e a de IGFBP-3, $-0,9 \pm 1,5$ (31,6\% abaixo de -2 DP).

\section{Estudo molecular}

Dezesseis dos 33 pacientes com SN $(48,5 \%)$ apresentavam mutações no PTPNIl: oito diferentes mutações missense já descritas e a mutação nova $\mathrm{P} 491 \mathrm{H}$ (tabela 2). Seis das mutações encontradas (67\%) localizam-se no exon 3 , que codifica o domínio N-SH2. As outras três situam-se nos exons 8 (11\%) e 13 (22\%), responsáveis pelo domínio PTP. Elas encontram-se em aminoácidos envolvidos diretamente na ligação intramolecular entre os domínios N-SH2 e PTP (N58D, G60A, D61N, Y63C, A72G, T73I) ou localizados em torno da superfície de interação desses domínios (N308D, P491H e M504V).

\section{Correlação do genótipo com o fenótipo}

Comparando os grupos com e sem mutação no PTPN11, observamos uma tendência não significativa do grupo com mutação de apresentar fácies típica com maior freqüência (75 vs. $47 \%$ ) (tabela 3 ). Foi observada uma maior freqüência de estenose pulmonar no grupo de pacientes com mutação em relação aos pacientes sem mutação $(38 \%$ vs. $6 \%, \mathrm{p}<0,05)$. Os dois grupos de pacientes não diferiram em relação à média do $\mathrm{Z}$ de altura, $\mathrm{Z}$ de IMC, freqüência de alterações torácicas, características faciais, criptorquidia, retardo mental, dificuldade de aprendizado, pico de $\mathrm{GH}$ ao teste de estímulo e Z de IGF-1 ou IGFBP-3 (tabela 2). Os pacientes com mutação no domínio N-SH2 $(n=9)$ e os pacientes com mutação no domínio $\mathrm{PTP}(\mathrm{n}=7)$ não diferiam nas características clínicas analisadas.

Cinco pacientes apresentavam a mesma mutação, N308D, e não diferiram em termos de fenótipo em relação aos demais. Entre si, esses pacientes apresentavam características bastante diversas com o $\mathrm{Z}$ da altura variando de $-3,2$ a $-1,1$, face variando de típica a sugestiva e alterações fenotípicas isoladas, como exo- ftalmo unilateral, estenose pulmonar e dificuldade de aprendizado.

\section{DISCUSSĀo}

A síndrome de Noonan é uma causa importante de baixa estatura, apresentando uma incidência igual ou maior à da síndrome de Turner, sendo, porém, provavelmente sub-diagnosticada. A dificuldade em diagnosticar a SN deve-se principalmente à grande variabilidade fenotípica, principalmente nos casos com características faciais leves com ausência de malformações cardíacas, e a falta de um marcador bioquímico ou molecular para confirmação da suspeita clínica. Com a descrição do gene PTPNII como responsável pela $\mathrm{SN}$, tornou-se possível a comprovação laboratorial do diagnóstico clínico em aproximadamente metade dos pacientes e vários estudos avaliando o diagnóstico e tratamento da $\mathrm{SN}$ têm sido publicados recentemente (6,10-15).

Para o endocrinologista, a SN tem importância por ser um dos diagnósticos diferenciais da baixa estatura; em nossa casuística, 79\% dos pacientes apresentavam baixa estatura. A causa da desaceleração do crescimento pós-natal nas crianças com SN ainda é desconhecida e não é explicada pela presença de malformações cardíacas (16). Como observado em nossa casuística, os pacientes com SN não apresentam deficiência de GH, porém podem apresentar concentrações séricas diminuídas de IGF-1 e IGFBP-3. É descrito que pacientes com SN apresentam nível médio de GH espontâneo noturno diminuído associado a nível baixo ou no limite inferior da normalidade de IGF-1, embora a resposta aos testes de estímulo seja habitualmente normal $(17,18)$. A resposta ao tratamento com hormônio de crescimento recombinante humano (hGH) em crianças com SN (19) é inferior à observada em crianças com deficiência de GH (20), em crianças nascidas pequenas para idade gestacional (2l) ou com baixa estatura idiopática (22), sugerindo que uma insensibilidade parcial ao GH seria a causa da baixa estatura desses pacientes.

A descrição de mutações no gene PTPNII em pacientes com SN permitiu, pela primeira vez, a obtenção de um marcador genético para essa síndrome. Em nosso estudo, identificamos em dezesseis indivíduos, quinze casos esporádicos e um familiar, mutações missense no PTPN11 em estado de heterozigose. A freqüência global de mutações foi de $48,5 \%$, similar à descrita na maioria dos estudos prévios (12,23-25). Descrevemos, também, uma mutação nova no códon 491, que troca o aminoácido prolina por 
Tabela 2. Mutações no PTPN11 identificadas em heterozigose em 16 pacientes com SN.

\begin{tabular}{|c|c|c|c|c|c|}
\hline $\begin{array}{l}\text { Número de } \\
\text { pacientes }\end{array}$ & Exon & $\begin{array}{c}\text { Troca de } \\
\text { nucleotídeo }\end{array}$ & Troca de aminoácido & Códon & Domínio \\
\hline 1 & 3 & 172: $A \rightarrow G$ & Asn (N) > Asp (D) & 58 & $\mathrm{~N}-\mathrm{SH} 2$ \\
\hline 2 & 3 & 179: $\mathrm{G} \rightarrow \mathrm{C}$ & Gly $(G)>$ Ala $(A)$ & 60 & $\mathrm{~N}-\mathrm{SH} 2$ \\
\hline 1 & 3 & 181: $G \rightarrow A$ & Asp (D) > Asn (N) & 61 & $\mathrm{~N}-\mathrm{SH} 2$ \\
\hline 1 & 3 & 188: $A \rightarrow G$ & $\operatorname{Tyr}(\mathrm{Y})>$ Cys $(\mathrm{C})$ & 63 & $\mathrm{~N}-\mathrm{SH} 2$ \\
\hline 3 & 3 & 215: $C \rightarrow G$ & Ala $(A)>$ Gly $(G)$ & 72 & $\mathrm{~N}-\mathrm{SH} 2$ \\
\hline 1 & 3 & 218: $\mathrm{C} \rightarrow \mathrm{T}$ & $\operatorname{Thr}(\mathrm{T})>$ Ile $(\mathrm{I})$ & 73 & $\mathrm{~N}-\mathrm{SH} 2$ \\
\hline 5 & 8 & 922: $A \rightarrow G$ & Asn (N) > Asp (D) & 308 & PTP \\
\hline 1 & 13 & 1477: $C \rightarrow A$ & Pro $(\mathrm{P})>\mathrm{Hls}(\mathrm{H})$ & 491 & PTP \\
\hline 1 & 13 & 1510: $A \rightarrow G$ & Met $(\mathrm{M})>$ Val $(\mathrm{V})$ & 504 & PTP \\
\hline
\end{tabular}

Asn= asparagina; Asp= ácido aspártico; Gly= glicina; Ala= alanina; His= histidina; lle= isoleucina; $\mathrm{Thr}=$ treonina; $\mathrm{Tyr}=$ tirosina; $\mathrm{Cys}=$ cisteína; Met= metionina; $\mathrm{Pro}=$ prolina; $\mathrm{Val}=\mathrm{valina}$.

Tabela 3. Comparação do fenótipo dos pacientes de SN com e sem mutação no PTPN11.

\begin{tabular}{lccc}
\hline Aspecto & $\begin{array}{c}\text { Com mutação } \\
\text { No/total (\%) }\end{array}$ & $\begin{array}{c}\text { Sem mutação } \\
\text { No/total (\%) }\end{array}$ & NS \\
Craniofaciais & $12 / 16(75)$ & $8 / 17(47)$ & NS \\
Face típica & $9 / 16(25)$ & $7 / 17(53)$ & NS \\
Face sugestiva & $8 / 16(50)$ & $9 / 17(53)$ & NS \\
Alterações do pavilhão auricular & $3 / 16(19)$ & $7 / 17(41)$ & NS \\
Ptose palpebral & $5 / 16(31)$ & $4 / 17(24)$ & NS \\
Epicanto & $6 / 16(38)$ & $5 / 17(30)$ & NS \\
Hipertelorismo & $9 / 16(56)$ & $12 / 17(70)$ & NS \\
Pescoço alado & $8 / 16(50)$ & $9 / 17(53)$ & p 0,05 \\
Face triangular & & & NS \\
Defeito cardíaco & $6 / 16(38)$ & $1 / 17(6)$ & NS \\
Estenose pulmonar & $1 / 16(6)$ & $2 / 17(12)$ & NS \\
Miocardiopatia hipertrófica & $2 / 16(13)$ & $1 / 17(6)$ & NS \\
Defeito septal & $11 / 16(69)$ & $15 / 17(88)$ & NS \\
Baixa estatura (Z altura <-2) & $-2,8 \pm 1,3$ & $-2,6 \pm 1,0$ & NS \\
Z altura & $-1,1 \pm 1,5$ & $-0,9 \pm 1,3$ & NS \\
Z IMC & $7 / 16(43,5)$ & $10 / 17(58,8)$ & NS \\
Alterações torácicas & $7 / 12(58)$ & $7 / 11(64)$ & NS \\
Criptorquidia & $7 / 16(43,5)$ & $7 / 17(41,2)$ & NS \\
Dificuldade de aprendizado & $11,5 \pm 6,9$ & $13,8 \pm 5,8$ & NS \\
Pico de GH (ng/ml) & $-1,2 \pm 1,3$ & $-1,9 \pm 2,0$ & $-0,8 \pm 2,0$ \\
Z de IGF-1 & $-1,0 \pm 0,9$ & & \\
Z de IGFBP-3 & & & \\
\hline
\end{tabular}

NS: Não significante

histidina $(\mathrm{P} 491 \mathrm{H})$; nessa mesma localização, outras duas mutações já foram descritas com a presença do aminoácido serina ou leucina nesta posição (P491S e P491L) (6). A causa genética da $\mathrm{SN}$ dos pacientes sem mutação do PTPNII ainda não está esclarecida, porém recentemente mutações nos genes KRAS foram descritas em 2,3\% dos pacientes com SN sem mutação no gene PTPNII (26) e em 7\% dos pacientes com síndrome cárdio-fácio-cutânea (CFC) (27), uma síndrome que compartilha características clínicas com a SN. Tanto a proteína K-Ras quanto a SHP-2 estão envolvidas na via de sinalização do Ras e, assim sendo, outras proteínas dessa cascata estão sendo investigadas como candidatas a causarem SN.

Comparando os grupos com e sem mutação no PTPN11, observamos que os pacientes com mutação apresentam maior freqüência de estenose pulmonar em concordância com estudos anteriores $(12,13,24)$. 
Além da estenose pulmonar, mutações no PTPN11 em pacientes com SN também foram associadas com a presença de baixa estatura mais intensa $(13,28)$ e maior freqüência de fácies típica (13).

Observamos uma grande variabilidade fenotípica entre os pacientes portadores de mutação. Os cincos pacientes que apresentavam a mutação N308D não apresentavam características que os diferenciassem dos outros pacientes, além de apresentarem características clínicas distintas entre si. A mutação N308D é uma das mais freqüentemente encontradas em pacientes com SN e está associada com os mais diferentes graus de gravidade da doença, desde face somente sugestiva até quadro muito típico com ou sem retardo mental e estenose da valva pulmonar $(13,24)$.

Recentemente, descrevemos o comportamento de pacientes com SN frente à terapia hGH em relação à presença ou ausência de mutação no gene PTPN11 (29). $\mathrm{O}$ tratamento com doses suprafisiológicas de hGH normalizou o nível de IGF-1 basal em ambos os grupos; entretanto, pacientes com mutação apresentaram menor aumento de IGF-l comparados com os pacientes sem mutação, mesmo utilizando dose semelhante de hGH (29). Apesar dos parâmetros clínicos semelhantes, pacientes sem mutação no gene PTPNII apresentaram tendência a melhor velocidade de crescimento, que se traduziu em significativo ganho de altura no terceiro ano de tratamento, fato que indica uma influência negativa das mutações na resposta ao tratamento com hGH (29). Este estado de insensibilidade parcial ao GH nos pacientes com SN com mutação no PTPNII foi comprovado por estudos subseqüentes $(25,28)$ e provavelmente é causado pelo efeito negativo na transdução do sinal do GH exercido pela SHP-2 mutada (7).

Em conclusão, a SN é uma causa importante de baixa estatura. Durante a investigação da baixa estatura, devemos suspeitar de SN em crianças com características faciais da síndrome e avaliá-las segundo os critérios de van der Burgt, que permitem seu adequado diagnóstico clínico. O estudo molecular do gene PTPNII possibilitará confirmação dessa síndrome em, pelo menos, 40-50\% dos pacientes, permitindo aconselhamento genético, melhor orientação do acompanhamento, além de identificar um subgrupo de pacientes com pior resposta ao tratamento com hGH.

\section{AGRADECIMENTOS}

Trabalho realizado parcialmente com auxílio financeiro da Fundação de Amparo Pesquisa do Estado de São Paulo (FAPESP) - Projeto temático número
00/14092-4. AALJ é bolsista FAPESP (\#02/096874). BBM e IJPA possuem auxílio do Conselho Nacional de Pesquisa (CNPq) número 031246/1995-5 e 303444/2002-9, respectivamente.

\section{REFERÊNCIAS}

1. Nora JJ, Nora AH, Sinha AK, Spangler RD, Lubs HA. The UIIrich-Noonan syndrome (Turner phenotype). Am J Dis Child 1974; 127:48-55.

2. Jones KL. Smith's Recognizable Patterns of Human Malformation. $5^{\text {th }}$ ed. Philadelphia: WB Saunders Co., 1997.

3. Ranke MB, Heidemann P, Knupfer C, Enders H, Schmaltz AA, Bierich JR. Noonan syndrome: growth and clinical manifestations in 144 cases. Eur J Pediatr 1988;148:220-7.

4. van der Burgt I, Berends $E$, Lommen $E$, van Beersum $S$, Hamel B, Mariman E. Clinical and molecular studies in a large Dutch family with Noonan syndrome. Am J Med Genet 1994;53:187-91.

5. Tartaglia M, Mehler EL, Goldberg R, Zampino G, Brunner HG, Kremer $\mathrm{H}$, et al. Mutations in PTPN11, encoding the protein tyrosine phosphatase SHP-2, cause Noonan syndrome. Nat Genet 2001;29:465-8.

6. Tartaglia M, Martinelli S, Stella L, Bocchinfuso G, Flex E, Cordeddu V, et al. Diversity and Functional Consequences of Germline and Somatic PTPN11 Mutations in Human Disease. Am J Hum Genet 2006; 78:279-90

7. Stofega MR, Herrington J, Billestrup N, Carter-Su C. Mutation of the SHP-2 binding site in growth hormone $(\mathrm{GH})$ receptor prolongs $\mathrm{GH}$-promoted tyrosyl phosphorylation of $\mathrm{GH}$ receptor, JAK2, and STAT5B. Mol Endocrinol 2000;14:1338-50.

8. Yamada S, Shiono S, Joo A, Yoshimura A. Control mechanism of JAK/STAT signal transduction pathway. FEBS Lett 2003;534:190-6.

9. Silva EG, Slhessarenko N, Arnhold IJ, Batista MC, Estefan V, Osorio MG, et al. GH values after clonidine stimulation measured by immunofluorometric assay in normal prepubertal children and GH-deficient patients. Horm Res 2003,59:229-33.

10. Lee JS, Tartaglia M, Gelb BD, Fridrich K, Sachs S, Stratakis $\mathrm{CA}$, et al. Phenotypic and genotypic characterisation of Noonan-like/multiple giant cell lesion syndrome. J Med Genet 2005;42:e11.

11. Kratz CP, Niemeyer CM, Castleberry RP, Cetin M, Bergstrasser E, Emanuel PD, et al. The mutational spectrum of PTPN11 in juvenile myelomonocytic leukemia and Noonan syndrome/myeloproliferative disease. Blood 2005;106:2183-5.

12. Jongmans $M$, Sistermans EA, Rikken A, Nillesen WM, Tamminga $R$, Patton $M$, et al. Genotypic and phenotypic characterization of Noonan syndrome: new data and review of the literature. Am J Med Genet 2005;134:165-70.

13. Zenker M, Buheitel G, Rauch R, Koenig R, Bosse K, Kress W, et al. Genotype-phenotype correlations in Noonan syndrome. J Pediatr 2004;144:368-74.

14. Yoshida R, Hasegawa T, Hasegawa Y, Nagai T, Kinoshita E, Tanaka $Y$, et al. Protein-tyrosine phosphatase, nonreceptor type 11 mutation analysis and clinical assessment in 45 patients with Noonan syndrome. J Clin Endocrinol Metab 2004;89:3359-64.

15. Tartaglia M, Cordeddu V, Chang H, Shaw A, Kalidas K, Crosby $A$, et al. Paternal germline origin and sex-ratio distortion in transmission of PTPN11 mutations in Noonan syndrome. Am J Hum Genet 2004;75:492-7.

16. Noonan JA, Raaijmakers R, Hall BD. Adult height in Noonan syndrome. Am J Med Genet 2003;123:68-71.

17. Ahmed ML, Foot $A B$, Edge JA, Lamkin VA, Savage $M O$, Dunger DB. Noonan's syndrome: abnormalities of the growth hormone/IGF-I axis and the response to treatment with human biosynthetic growth hormone. Acta Paediatr Scand 1991;80:446-50. 
18. Noordam C, van der Burgt I, Sweep CG, Delemarre-van de Waal HA, Sengers RC, Otten BJ. Growth hormone (GH) secretion in children with Noonan syndrome: frequently abnormal without consequences for growth or response to GH treatment. Clin Endocrinol (Oxf) 2001;54:53-9.

19. Romano AA, Blethen SL, Dana K, Noto RA. Growth hormone treatment in Noonan syndrome: the National Cooperative Growth Study experience. J Pediatr 1996;128:S18-21.

20. Ranke MB, Lindberg $A$, Chatelain $P$, Wilton $P$, Cutfield $W$ Albertsson-Wikland $K$, et al. Derivation and validation of a mathematical model for predicting the response to exogenous recombinant human growth hormone $(\mathrm{GH})$ in prepubertal children with idiopathic GH deficiency. KIGS International Board. Kabi Pharmacia International Growth Study. J Clin Endocrinol Metab 1999;84:1174-83.

21. Ranke MB, Lindberg A, Cowell CT, Wikland KA, Reiter EO, Wilton $\mathrm{P}$, et al. Prediction of response to growth hormone treatment in short children born small for gestational age: analysis of data from KIGS (Pharmacia International Growth Database). J Clin Endocrinol Metab 2003;88:125-31.

22. Finkelstein BS, Imperiale TF, Speroff T, Marrero U, Radcliffe DJ, Cuttler L. Effect of growth hormone therapy on height in children with idiopathic short stature: a meta-analysis. Arch Pediatr Adolesc Med 2002; 156:230-40.

23. Maheshwari M, Belmont J, Fernbach S, Ho T, Molinari L, Yakub I, et al. PTPN11 mutations in Noonan syndrome type detection of recurrent mutations in exons 3 and 13. Hum Mutat 2002;20:298-304.

24. Tartaglia M, Kalidas K, Shaw A, Song X, Musat DL, Van Der Burgt I, et al. PTPN11 mutations in Noonan Syndrome: molecular spectrum, genotype- phenotype correlation, and phenotypic heterogeneity. Am J Hum Genet 2002;70:1555-63.
25. Binder G, Neuer K, Ranke MB, Wittekindt NE. PTPN11 mutations are associated with mild growth hormone resistance in individuals with Noonan syndrome. J Clin Endocrinol Metab 2005;90:5377-81.

26. Schubbert S, Zenker M, Rowe SL, Boll S, Klein C, Bollag G, et al. Germline KRAS mutations cause Noonan syndrome. Nat Genet 2006:38:331-6.

27. Niihori T, Aoki Y, Narumi Y, Neri G, Cave H, Verloes A, et al. Germline KRAS and BRAF mutations in cardio-facio-cutaneous syndrome. Nat Genet 2006;38:294-6.

28. Limal JM, Parfait B, Cabrol S, Bonnet D, Leheup B, Lyonnet S, et al. Noonan syndrome: relationships between genotype, growth, and growth factors. J Clin Endocrinol Metab 2006; $91: 300-6$.

29. Ferreira LV, Souza SA, Arnhold IJ, Mendonça BB, Jorge AA. PTPN11 (protein tyrosine phosphatase, nonreceptor type 11) mutations and response to growth hormone therapy in children with Noonan syndrome. J Clin Endocrinol Metab 2005;90:5156-60.

Endereço para correspondência:

Alexander A.L. Jorge

Av. Dr. Enéas de Carvalho Aguiar 155, 2 andar, Bloco 6

05403-900 São Paulo, SP

Fax: (11) 3069-7519

E-mail: alexj@usp.br 